\title{
LXXVIII.
}

\section{Eine neue Methode, die Menge der salpeter- sauren Salze und besonders des Salpeters zu bestimmen.}

\author{
Von \\ Pelouse. \\ (Compt. rend. XXIV, p. 209.)
}

Da alle salpetersauren Salze in Wasser löslich sind, so kann man zu ihrer Bestimmung nicht die gewöhnlich für die andern Salze gebräuchliche Methode benutzen, welche darin besteht, einen Niederschlag von bekannter Zusammensetzung zu bilden, welcher ausgewaschen, getrocknet und gewogen wird.

Indessen, da die Anwendung des Salpeters sebr beträchtlich ist, indem die Fabrication des Pulvers selbst in Friedenszeiten jährlich mehrere Millionen Kilogramme Salpeter erfordert, so hat man seit langer Zeit Mittel gesucht, um, wenn auch nicht eine auf ganz scharfe, doch eine hinreichend annähernde Weise den Werth des rohen Salpeters zu bestimmen, welcher in den öffentlichen Fabriken raffinirt werden soll.

Vor 1775, der Zeit, als die Pulverfabrication Regie wurde, kannte man keine Methode, selbst keine rohe, um den Salpeter zu prüfen. Man begnügte sich damit, eine gewisse Quantität desselben in einem eisernen Löffel zu glühen, um durch das $A b$ brennen und Verknistern die relativen Mengen von Salpeter und Kochsalz zu bestimmen.

Im Jahre 1783 schlug Guyto n de Morvea ein analytisches Mittel vor, welches in der aufeinanderfolgenden Anwendung des Alkohols und des salpetersauren Bleioxyds bestand. Dieses noch sehr unvollkommene und dabei schwierig auszuführende Verfahren wurde allein in der Raffinerie von Paris angewendet, wo die Proben unter den Augen Lavoisier's angestellt wurden. Die Resultate der Proben stimmten nicht mit 
den Producten der Raffinage uiberein; B eaumé substituirte dem Bleinitrat das Acetat, jedoch ohne wirklichen Vortheil.

1789 glaubte man endlich den Zweck erreicht zu haben, den man so lange gesucht hatte. Riffault hatte die ingenieuse Idee, den Alkohol durch eine gesăttigte Salpeterlösung zu ersetzen. L a vo isier nahm diese Idee auf und verlieh ihr die Stütze seiner grossen Autorităt; jedoch geleitet, wie er selbst sagt, durch einen Versuch von Ge offroy, zeigte er, dass in dem Riffault'schen Verfabren eine Ursache zu einem Irrthume liege, welche darin besteht, dass das Chlornatrium, zum Schaden des Lieferanten, die Auflösung einer beträchtlichen Menge Salpeters in der Probe bewirkt.

Man entwarf daher eine Correctionstabelle, in welcher man die Wirkung der.in der Probe gefundenen Salzmenge zu bestimmen suchte; jedoch waren auch jetzt noch nicht die Producte der Raffinerie mit der Probe in Uebereinstimmung.

Die Academie der Wissenschaften, von dem Gouvernement consultirt, nahm auf den Vorschlag von Berthollet und Fourcroy das Verfahren an, welches von der Administration der Pulverfabriken, zu der Lavo isier gehörte, angegeben und ausgearbeitet war.

Ungeachtet dieses günstigen Berichtes und der grossen Autorităt der Chemiker, welche bei seiner Abfassung thătig gewesen waren, kamen von allen Seiten Klagen über die Ungenauigkeit des Riff a ult'schen Verfahrens.

Am 27. Juli 1791 lasen Fourcroy und Vauquelin eine Denkschrift über die Ungenauigkeiten und die Fehlerquellen des Probe-Verfahrens mit der gesăttigten Salpeterlösung, und, weit entfernt, den Klagen der Salpeterfabricanten. Recht zu geben, machten sie den Vorschlag, die nach der, der Instruction vom 1. Juli 1789 beigefügten Tabelle zugestandene Entschädigung zu unterdrücken. Der Minister, zwischen diesen Berichten und den Forderungen, die so entgegengesetzt lauteten, schwankend, wendete sich von Neuem an die Academie, welche zur Prüfung dieser Angelegenheit eine Commission ernannte, die aus B eaumé, Berthollet, d'Arcet, Vater, und Fourcroy zusammengesetzt war. Die Commissaire, denen V a u qu e lin noch hinzugefügt wurde, erklärten, dass, ungeachtet einiger leichten Ungenauigkeiten, die Probe mit der gesättigten Salpeterlösung diejenige sei, welche 
durch ihre Einfachheit den grössten Vorzug verdiene. Sie wiesen die Correctionen in Beziehung auf den Einfluss des Kochsalzes zurück.

Zu dieser Zeit erregte Alles, was mehr oder weniger direct das Pulver belraf, in hohem Maasse das Interesse des Publicums. Die Assemblée nationale gab am 14. Mai 1792 ein Decret, dass der Minister der öffentlichen Steuern, zusammen mit der Verwaltung der Pulver- und Salpeterfabriken und der Academie der Wissenschaften, ihr einen Vorschlag zu einem Reglement einreichen sollten für die Form der Abnahme des Salpeters und für die Prüfung des Rückstandes desselben.

L a v o i sie r wurde speciell beauftragt mit Versuchen, welche zur Grundlage des Entwurfes dienen sollten. Wie er uns selbst mittheilt, beschăftigte er sich ohne Unterlass wăhrend der vier Monate damit, welche seinem Austritt aus der Verwaltung der Pulverfabrik vorausgingen. Seine Arbeiten, wichtig für die Fabrication und Raffinage des Salpeters, haben dennoch nicht zur vőlligen Lösung des Problems über die Analyse dieses Salzes geführt.

Zu dieser Zeit wurde der Verkehr zwischen Frankreich und Indien unterbrochen; man musste ganz unumgänglich allen Salpeter, den man zu seiner Vertheidigung und zur Versorgung der zahlreichen Armeen verbrauchte, allein aus dem heimischen Boden gewinnen. Die Regie verschwand; eine revolutionäre Verwaltung trat an ihre Stelle; die Städte, die Gemeinden, Privaten beschäftigten sich mit der Salpeterfabrication. Wie auch der Gehalt des Productes war, man häufte es, obne eine Prüfung, in den National-Magazinen auf, und die Verantwortlichkeit trat erst wieder ein, als Frankreich seine Unabhängigkeit gesichert hatte.

Von Neuem wurde die Academie um die beste Salpeterprūfung befragt. Pelletier, Vauquelin, Guytonde Morveau, als Berichterstatter, arbeiteten zusammen mit $\mathrm{Champy}$ eine Instruction aus, welche vom Institut am 11. Messidor des Jahres $\mathbf{V}$ gebilligt und von dem ausũbenden Directorium am 1. Vendemiaire VI (22. September 1797) für bindend erkłărt wurde. Diese Instruction, bis zum heutigen Tage im Gebrauch, ist fast vollstăndig die Riffault'sche Methode, welche durch Lavoisier sanctionirt war, 
Ein wirklich eigenthümlicher Umstand ist der, dass in keiner der Arbeiten, deren Gegenstand die Salpeterprüfung war, jemals die Rede von dem störenden Einflusse ist, welchen die häufige Gegenwart des Chlorkaliums in dem Salpeter hervorrufen kann. - Dieser Einfluss wurde 1815 erst angegeben. Zu dieser Zeit wies der Commissarius der Pulverfabrik zu Lille ein beträchtliches Deficit nach, dessen Ursache man auffand, als man die Salze untersuchte, welche während der Reinigung vom Salpeter abgeschieden worden waren. Diese Salze, welche man für Kochsalz hielt, bestanden fast gänzlich aus Chlorkalium.

Das Deficit erklärte sich leicht durch eine zu hole Annahme des Werthes des Salpeters, was daher kam, dass das Chlorkalium in einer gesättigten Salpeterlösung niederfällt.

Saint-Venant und Pelissier und andrerseits Mallet und Perruché beschäftigten sich damit, Mittel aufzufinden, die Gegenwart des Chlorkaliums in dem rohen Salpeter zu entdecken, und suchten seinen Einfluss auf die angenommene Prüfungsmethode zu bestimmen. Ohne in der Instruction etwas zu ändern, befahl die Pulverdirection den Commissären (5. Aug. 1820), keinen Salpeter anzunehmen, welcher mehr als 15 p. C. Abgang erlitt. 1829 arbeitete $G$ a $y-L u s s$ a cine Instruction aus, in welcher er die Correctionen angab, denen man das Resultat der Proben unterwerfen muss; sie war gegründet auf die Temperaturerniedrigung, welche (beim Auflösen des Salpeters) durch das Chlorkalium, oder vielmehr durch ein Gemenge desselben und des Kochsalzes, hervorgebracht wird.

Endlich untersuchte Fauché den Einfluss, welchen die in dem rohen Salpeler enthaltenen Salze auf die Löslichkeit desselben ausüben; er suchte den Irrthum zu beseitigen, den das Chlorkalium bei den Proben dieses Salzes herbeigeführt.

Um das Geschichtliche der Arbeiten über diesen Gegenstand zu beenden, muss ich noch von einer Notiz sprechen, die Herr Gossart, Commissaire eles poudres zu Lille, der Academie mitzutheilen mich gebeten; sie ist abgedruckt in den Comptes rendus No. 1, 1847. Hr. Gossart suchte den Grad der Reinheit des rohen Salpeters dadurch zu bestimmen, dass er das Salz mit Schwefelsäure mischte und es durch eine titrirte Lòsung von Eisenvitriol zerlegte. Er hält die Operation für beendet, wenn er nach dem Erhitzen der Flüssigkeit, eine kleine Menge davon, 
abgeschieden hat und in dieser durch Kaliumeisencyanid die Gegenwart von Eisenoxydul findet.

Die Methode von Gossart ist auf das Princip gegründet, welches Gay-Lussac und mehrere andere Chemiker für die Chlorometrie und die Braunsteinprobe angewendet haben.

Ich habe mich gleichfalls mit der Prüfung der Bestimmung des Salpeters beschäftigt und musste daher die Versuche von Gossart wiederholen. Ich glaubte einige Unvollkommenheit in dem Verfahren zu erkennen, was ich dem Verfasser auch mitgetheilt habe; er beauftragte mich, der Academie eine neue Note vorzulegen, in der er eine Umänderung seines Verfahrens auseinandersetzt.

Ich komme nun $z u$ den Versuchen, welche mich $z u$ dem Verfahren geführt haben, welches ich für die Analyse der Nitrate vorschlage.

Zu den Metallen, welche sich mit Leichtigkeit höher oxydiren oder chloren, gehört, wie man weiss, das Eisen. Man weiss, dass nach den Versuchen von Margueritte eine verdünnte Eisenoxydullösung (oder eigentlich Eisenchlorürlösung) sich augenblicklich höher oxydirt, wenn man bei gewöhnlicher Temperatur eine Auflösung von übermangansaurem Kali hinzufügt, und dass die kleinste Menge von mineralischem Kamäleon, welche man der so höher oxydirten Eisenlösung hinzufügt, der Flüssigkeit eine Rosafarbe ertheilt, welche ein sicheres Kennzeichen für den Augenblick der vollendeten Oxydation wird.

Ich habe gesucht, auf sehr genaue Weise zu bestimmen, wie viel salpetersaures Kali erfordert wird, um ein gegebenes Gewicht Eisen, welches in Chlorwasserstoffsäure gelöst war, auf das Maximum der Oxydation zu bringen. Ich wăhlte, der Bequemlichkeit halber, Claviersaiten, welche man bis auf $2-3$ Tausendtel als reines Eisen betrachten kann. Ich fand, dass $2 \mathrm{Grm}$. dieses Eisens, gelöst in einem beträchtlichen Ueberschuss von Chlorwasserstoffsäure $(80-100 \mathrm{Grm}$.), um in Chlorid verwandelt zu werden, zwischen 1,212 und 1,220 Grm. erforderten, im Mittel 1,216 Grm. Salpeter. Ich habe das Gas untersucht, welches bei dieser Reaction entwickelt wird, und gefunden, dass es aus Stickoxyd, gemischt mit Chlorwasserstoffsäure, besteht.

Indem man die Zahlen in die Aequivalentzahlen übersetzt, sieht man, dass 6 Aeq. Eisen auf 1 Aeq. Salpeter kommen. 
Die Säure des letztern spaltet sich in Stickoxyd, welches entweicht, und 5 Aeq. Sauerstoff, welche 3 Aeq. Wasserstoff aufnehmen, um 3 Aeq. Chlor an das Eisenchlorür treten zu lassen, so dass 3 Aeq. Eisenchlorid entstehen.

$$
6 \mathrm{Fe}+6 \mathrm{Cl} \mathrm{H}=6 \mathrm{FeCl}+6 \mathrm{H} \text {. }
$$

$\left.6 \mathrm{FeCl}+\mathrm{NO}_{5}, \mathrm{KO}+4 \mathrm{ClH}=4 \mathrm{HO}+\mathrm{KCl}+\mathrm{NO}_{2}+3 \mathrm{Fe}_{2} \mathrm{Cl}_{3}{ }^{*}\right)$.

Da diese Reaction so einfach vor sich geht, so konnte ich leicht vorhersehen, dass sie die Basis für eine einfache Methode, die Nitrate zu analysiren, bilden würde.

Es war nicht zu vermuthen, dass die Gegenwart von Chlorüren und Sulphaten in den zu prüfenden Salzen, und namentlich im Salpeter," die Art und Weise jener Zersetzung würde ändern können; jedenfalls jedoch musste ich mich durch directe Versuche davon unterrichten und fügte daher zu dem reinen Salpeter eine mehr oder minder bedeutende Menge von Kochsalz, Chlornatrium, schwefelsaurem Natron und Kali; ich fand, dass alle diese Salze ohne Einfluss auf die oxydirende Wirkung des Salpeters auf das Eisen seien.

Es blieb mir noch übrig, ein sicheres und einfaches Verfahren aufzusuchen, bei der Prüfung des unreinen Salpeters die Menge des nicht oxydirten Eisens zu bestimmen. Das Verfahren dazu ist das, welches Margueritte in seiner Abhandlung bereits angegeben ${ }^{* *}$ ).

Vorausgesetzt, dass man mit $2 \mathrm{Grm}$. Eisen und 1,216 Grm. unreinem Salpeter durch das mineralische Kamäleon gefunden, dass 0,200 Grm. Eisen nicht in den Zustand des $0 x y d s$ versetzt worden waren, so sind also nur 1,800 Grm. Eisen so weit oxydirt worden, während bei ganz reinem Salpeter die $2 \mathrm{Grn}$. vollständig in das Chlorid würden verwandelt worden sein. Man hat also die Proportion:

$$
\begin{aligned}
& 2,000: 1,216=1,800: x, \\
& x=1,0944 .
\end{aligned}
$$

In 1,210 Grm. rohem Salpeter sind also wirklich nur 1,0944 Grm. reiner Salpeter enthalten gewesen, oder 90 p. C.

*) Diese Zersetzung der salpetersauren Salze durch die Eisenoxydulsalze bei Gegenwart von einem Ueberschuss an Salzsäure liefert ein vortreffliches Mittel fïr die Darstellung des Stickstoffoxydes.

**) Dies. Journ. XXXVIII, S. 160. 
In einen Kolben von $150 \mathrm{Cb}$. C. Inhalt ungefähr bringt man 2 Grm. Claviersaiten und schūttet darauf 80-100 Grm. concentrirter Chlorwasserstoffsäure; der Kolben wird durch einen Kork geschlossen, welcher mit einer in eine Spitze ausgezogenen offenen Glasröhre versehen ist, worauf man das Eisen bei gelinder Wărme auflöst. Sowie die Auflösung erfolgt ist, bringt man 1,200 Grm. des Salpeters, der zu prüfen ist, hinein; sogleich wird der Kolben geschlossen und bis zum Kochen erhitzt. Die Flüssigkeit bräunt sich stark; dicke Dämpfe von Chlorwasserstoffsäure, beladen mit Stickstoffoxyd, entweichen durch die Spitze mit Heftigkeit und hindern den Zutritt der Luft. Bald verliert die Flüssigkeit ihre braune Farbe; sie wird gelb, klärt sich auf; während die Masse fünf bis sechs Minuten gekocht hat und nachdem sie völlig durchsichtig geworden, zieht man sie vom Feuer und schüttet sie, nebst dem Spülwasser, mit dem man den Kolben sorgfältig reinigt, in einen Ballon von etwa 1 Lit. Inhalt, welchen man fast völlig mit gewöhnlichem Wasser füllt. Sodann fügt man mit einer graduirten Burette eine titrirte Lösung von äbermangansaurem Kali hinzu. Durch eine Bewegung des Kolbens mischt man die Flüssigkeiten; wenn diese eine leichte Rosafarbe annehmen, hört man auf, von dem Kamäleon binzuzusezzen, und liest an der Burette $a b$, wie viel man von der Probeflüssigkeit gebraucht hat*). Nehmen wir an, die Stărke des Kamäleons sei so, dass man $50 \mathrm{Cb}$.C. der Flüssigkeit brauchte, um $1 \mathrm{Grm}$. Eisen zu oxydiren, und, um den vorhergedachten Versuch zu beenden, wir $10 \mathrm{Cb}$.C. davon anzuwenden hätten, so hätten wir folgende Proportion:

50 Cb.C. $: 1,000=10$ Cb.C. $: 0,200$.

*) Man stellt des Kamäleon dar, indem man in einem irdenen Tiegel 3 Th. Aetzkali, 2 Th. Braunstein (fein gepulvert), 1 Th. chlorsaures Kali bei Dunkelrothglühhitze einige Zeit erhitzt. Die dunkelgrūne Masse wird gepulvert, mit dem Drei - oder Vierfachen ihres Gewichts Wasser gemengt, mit sehr schwacher Salpetersäure behandelt, bis sie purpurroth geworden, durch Asbest filtrirt und in einer Flasche mit eingeschliffenem Stopsel aufbewahrt.

Um den Gehalt der Flüssigkeit zu bestimmen, wiegt man genau 0,500 Grm. Claviersaite ab, welche in $15-20 \mathrm{Grm}$. Salzsäure gelost werden, vermischt die Flüssigkeit mit 1 Lit. Wasser und fügt so lange hinzu, bis eine Rosa-Fürbung der Flässigkeit eintritt. 
Es sind also von $2,000 \mathrm{Grm}$. Eisen $0,200 \mathrm{Grm}$. abzuziehen. Die 1,800 sind daher durch 1,200 des rohen Salpeters auf das Mlaximum der 0xydation gebracht. Da nun $2 \mathrm{Grm}$. Eisen 1,216 Grm. Salpeter erfordern, oder $1 \mathrm{Grm}$. Eisen 0,608 Grm. $\mathrm{NO}_{5}$, K 0 repräsentirt, so hat man :

$$
1,000: 0,608=1,800: x ; x=1,0944 \text {. }
$$

Die 1,200 Grm. des Salzes, welches der Prüfung unterworfen war, enthielten also 1,0944 Salpeter oder $\frac{1,0944}{1,2000}=91,2$ p.C.

Man sieht, dass bei dem Versuche die Einwirkung der Luft ausgeschlossen werden muss; hat dieselbe Zutritt zu dem Kolben, so muss ein Theil des Stickstoffoxyds in salpetrige Säure übergehen und auf diese Weise eine neue Menge Eisen höher oxydiren; der Werth des Salpeters würde daher überschätzt werden. Es ist indessen sehr leicht, diesen Uebelstand zu vermeiden. Wenn das Eisen in Kolben sich so eben gelöst hat, so ist derselbe angefüllt mit Wasserstoff und Chlorwasserstoffgas; der Salpeter, den man einbringt, führt höchstens eine Spur Luft mit sich, und die Flüssigkeit, bis zum Sieden erhitzt, lässt durch die Spitze fortwährend Dämpfe ausstrōmen, deren sichtbarer Strom leicht $\mathrm{zu}$ unterhalten ist und jeden Luftzutritt abwehrt.

Die Luft ist übrigens nur in dem Augenblick zu fürchten, wenn das salpetersaure Salz hinzugefügt wird, denn ich habe mich von der Richtigkeit der Angabe Marguerit te's überzeugt, welcher sagt, dass in einer stark sauren Flüssigkeit das Eisen sich an der selbst freien Luft so schwierig und so langsam oxydirt, dass die Analyse dadurch auf keine Weise beeinträchtigt wird.

Die salpetersauren Salze können im festen und im aufgelösten Zustande angewendet werden.

Im Allgemeinen ziehe ich die erste Form vor; wo es sich jedoch darum handelt, rohen Salpeter, der in den einzelnen Proben sehr verschieden zusammengesetzt sein kann, zu untersuchen, ist es vorzuziehen, eine Auflösung einer sehr grossen. Menge des Salzes zu benutzen. In diesem Falle verfahre ich auf folgende Weise :

Ich lōse in einem Gefâss von 2 Lit. Inhalt $120 \mathrm{Grm}$. Salpeter, oder in einem von 1 Lit. $60 \mathrm{Grm}$. Salpeter auf, indem ich das Gefăss gänzlich mit Wasser anfülle. Mit einer Pipette nehme ich 20 Cub.C. der Flüssigkeit und bringe sie in die Auf- 
Jösung von $2 \mathrm{Grm}$. Eisen in $100 \mathrm{Grm}$. Chlorwasserstoffsäure in dem Augenblick, wo das Metall verschwindet, und verfahre eben so wie mit dem krystallisirten Salpeter. Es ist klar, dass 20 Cub. C. einer solchen Flüssigkeit 1,200 rohen Salpeter darstellen, es ist also dieselbe Menge, mit welcher man bei dem trocknen Salze operirte.

Demselben Versuche unterwarf ich das salpetersaure Natron im reinen und unreinen Zustande; durch eine grosse Menge von Versuchen, deren Bericht unnũtz wãre, habe ich mich überzeugt, dass mein Verfahren gestattet, die Reinheit desselben mit grosser Genauigkeit zu prüfen.

Die Anwendung des salpetersauren Natrons scheint sich seht ausdehnen zu wollen; es wird benutzt zur Darstellung der Salpetersäure, des Salpeters und der Schwefelsäure; Kuhlmann hat seine glückliche Anwendung in der Agricultur kennen gelehrt. Es ist daher von Wichtigkeit, ein Mittel kennen zu lernen, es mit Schnelligkeit $\mathrm{zu}$ analysiren.

Das salpetersaure Ammoniak und das salpetersaure Bleioxyd haben mir mit meinem Verfahren Resultate geliefert, deren Interesse allein in der Bestätigung der Brauchbarkeit meiner Methode lag.

Diese Methode wird ein noch mehr praktisches Interesse gewähren für die Bestimmung der Wasserquantitäten, welche in manchen Nitraten nur unvollkommen gekannt sind. Sie wird Anwendung finden für die Prüfung des Säure-Gemisches aus Schwefelsäure und Salpetersãure, ehe man dasselbe zur Darstellung des Pyroxylins verwendet. Ich habe mich überzeugt, dass die Schwefelsäure, gemischt mit der Salpetersäure, durchaus keine Störungen für das neue Verfahren gewährt; man kann letztere Säure ganz eben so analysiren, mit Wasser verdünnt oder mit Schwefelsãure gemischt, wie Salpeter.

Die Salpeterfabricanten haben kein Mittel, mit einiger Genauigkeit die Menge der Nitrate zu bestimmen, welche in salpetersaures Kali verwandelt werden sollen. Sie können jetzt ihre Mauerabfälle und andere Salpeterstoffe mit Genauigkeit titriren*).

*) Es können jedoch Fälle vorkommen, in denen organische Stoffe, oder andere, welche der 0xydation fähig sind, sich den salpetersauren Salzen beigemischt finden und auf das Kamäleon wirken. 
Die Fabrication des Salpeters mit den Mauerabfällen geschieht hăufig auf die Weise, dass zu den hinreichend eingeengten Laugen schwefelsaures Natron gesetzt wird; diess giebt mit dem salpetersauren Kalk schwefelsauren Kalk, welcher sich absetzt, und salpetersaures Natron, welches gelöst bleibt; diess verwandelt man durch Chlorkalium in salpetersaures Kali. Wenn die Fabricanten die Menge der Salpetersäure in dieser Flüssigkeit kennen, so werden sie sicher geleitet werden für die Mlenge des anzuwendenden schwefelsauren Natrons; diess ist von grosser Wichtigkeit, da ein Mangel oder ein Ueberschuss dieses Salzes gleich schädlich wirkt.

Vorzugsweise ist es die Bestimmung des rohen Salpeters, für welche meine Methode wichtig sein wird. In den meisten Fällen enthält der Salpeter des Handels nur ein salpetersaures Salz, das Kochsalz, gemengt mit erdigen Bestandtheilen, Sulphaten und alkalischen Chlorüren. Der, welcher aus Indien kommt und welcher (in Frankreich) etwa $\frac{2}{3}$ der Menge ausmacht, welche für die Pulverfabrication bestimmt ist, ist vorzugsweise so zusammengesetzt. Jedoch lehrt natürlich das von mir angewendete Verfahren nicht die Basen kennen, welche mit der Salpetersäure verbunden sind; man findet immer nur die Menge der letzteren, so dass man sich immer überzeugen muss, ob nicht noch eine andere Basis mit der Salpetersăure verbunden ist. - So ist es z. B. sehr leicht zu bestimmen, die Menge des Salpeters, gemischt mit schwefelsauren Salzen oder Chlorüren; wenn aber salpetersaures Natron im Gemenge sich befindet, so findet man eben nur die Sāure, verbunden mit einem unbekannten Verhältniss beider Basen. Man wendet dann das Verfahren der Analyse an, welches Gay-Luss ac vorgeschlagen hat und welches darin besteht, die Nitrate durch Kohle zu zerlegen und mit einer Normalflüssigkeit von Schwefelsäure die Menge der resultirenden Carbonate zu bestimmen.

Es ist klar, dass man mein Verfahren nicht der jetzt angewendeten Salpeterprobe ausschliesslich substituiren darf, welches den Werth dieses Salzes, wenn auch nicht auf genaue Weise kennen lehrt, doch wenigstens innerhalb der Grenzen, welche einer billigen Abrechnung zwischen dem Staat und den Fabricanten genügen.

Die Methode, den Salpeter zu prüfen, besteht in den Raffinerien des Gouvernements darin, dass $400 \mathrm{Grm}$. Salpeter mit 
750 Cub.C. einer-gesăttigten, reinen Salpeterlösung gewaschen werden. Sie bietet einige Unsicherheiten dar, indem die erdigen Bestandtheile im Salpeter zurückbleiben; sie betragen meist 1-2 p. $\mathrm{C}_{\text {; }}$; und andrerseits die fremden Salze, namentlich die Sulphate und Chlorüre der Alkalien, mehr oder weniger auf die Probe und die gesăttigte Lösung wirken. Diese Unsicherheiten hāngen ausserdem von den Schwankungen der Temperatur $a b$, welche man zwar, aber doch nur unvollhommen, corrigirt, indem man untersucht, wie viel $400 \mathrm{Grm}$. reiner Salpeter unter denselben Umstānden durch 750 Cub.C. der gesättigten Lösung an Gewicht verlieren oder zunebmen. Wenn zufällig, was von Zeit zu Zeit eintritt, salpetersaures Natron im Salpeter enthalten ist, so würde es, wie die andern Salze, durch die gesăttigte Lơsung fortgeführt werden, und dem Fabricanten würde von der Regie kein Ersatz dafür geboten werden, da sie nur das Kalisalz von ihm kaufen will. Da nun das Natronsalz im letztern Falle mit dem Kalisalz verwechselt werden würde, wenn man mein Verfahren allein anwendete, so ist, wie ich schon sagte, klar, dass das Verfahren den Zweck hier nicht erfüllt, den man verfolgt; aber wenn sich dieser Uebelstand, dessen Wichtigkeit ich nicht verkenne, darbietet, so ergiebt sich glücklicher Weise dadurch eine unendlich grössere Genauigkeit, als das gebräuchliche Verfahren gewăhrt, wenn es sich darum handelt, die Menge des Salpeters zu bestimmen in einem Gemenge dieses Salzes und der Stoffe, welche es gewöbnlich begleiten. Es ist kein Zweifel, dass das neue Verfahren, mit dem alten verglichen, der Pulververwaltung grosse Dienste leisten wird. Ich habe durch dasselbe mehr, als man es früher konnte, die Schärfe der Riffault'schen Methode prüfen können.

Die vergleichende Analyse mehrerer unreiner Salpeterproben, welche zur Raffinage bestimmt waren, wurde mit der grössten Sorgfalt nach der alten und nach meiner Methode ausgeführt. Ich habe mich überzeugt, dass im grŏssten Theil der Fälle das Riffault'sche Verfahren einen zu hohen Werth giebt; die Differenz ist zuweilen bis auf 2-3 p. C. gestiegen. Dieser Umstand muss berücksichtigt werden; nach der Instruction fügt die Regie dem Abgange, welcher durch die Salpeterprobe gefunden ist, noch 2 p. C. hinzu; mit andern Worten, sie verringert den Werth des Salpeters um 2 p. C. Wenn dieser Gebrauch, seit 
Cahours: Ueberdie Schwefelverbindungen etc. $\quad 335$

einem halben Jahrhundert befolgt, voraussetzen lässt, dass das durch die Administration angenommene Verfahren sehr unvollkommen ist von dem analytischen Gesichtspuncte aus, so zeigt sich doch darin, dass die ausgezeichneten Männer, welche zu den verschiedensten Zeiten sich mit dieser Frage beschäftigt haben, ein billiges Verhältniss zwischen dem Interesse des Schatzes und. dem der Fabricanten herzustellen wussten. Das neue Verfahren wird von grossem Nutzen für die Pulververwaltung sein, nicht allein bei der Prüfung des Salpeters, welcher frei von andern Nitraten ist, sondern auch für den, welcher mit salpetersaurem Natron verbunden ist. Wenn die Methode einen höheren Werth giebt als die Riffault'sche, und die Differenz übersteigt 3 p.C., so wird man zu sehen haben, ob diess nicht durch die Beimischung des salpetersauren Natrons herbeigeführt wird.

Die neue Methode bietet, wenn es sich um ein Nitrat mit bekannter Basis handelt, den Vortheil einer leichten Ausführung dar; sie erfordert kaum 20 Minuten und gewährt eine Genauigkeit von 2-3 Tausendtel.

Um mich von dem Grade der Genauigkeit zu vergewissern, habe ich reinen Salpeter abgewogen und mit Sulphaten und Chlorüren gemengt. Die Analyse wurde nun ausgeführt und erreichte eine Uebereinstimmung mit der abgewogenen Menge, welche selten bis auf 5 Tausendtel differirte, gewöhnlich auf nur 2-3 Tausendtel.

\section{LXXIX. \\ Ueber die Schwefelverbindungen des Methyls. \\ Von. \\ Cothours.}

(Ann. de Chim. et de Phys. T. XIX. Féor. 1847, p. 158.)

In seiner kürzlich veröffentlichten Auhhandlung hat $\mathrm{Z}$ e is e gezeigt, dass das Jod, indem es auf eine weingeistige Lõsung von xanthogensaurem. Kali einwirkt, dieses Salz zersetzt und ein 